\title{
Knowledge silos: assessing knowledge sharing between specialties through the vestibular schwannoma literature
}

\author{
Zane Schnurman, BA, ${ }^{1}$ John G. Golfinos, MD, ${ }^{1}$ J. Thomas Roland Jr., MD, ${ }^{2}$ and \\ Douglas Kondziolka, MD'
}

\begin{abstract}
Departments of ${ }^{1}$ Neurosurgery and ${ }^{2}$ Otolaryngology Head and Neck Surgery, NYU Langone Medical Center, New York, New York
\end{abstract}

\begin{abstract}
OBJECTIVE It is common for a medical disorder to be managed or researched by individuals who work within different specialties. It is known that both neurosurgeons and neurotologists manage vestibular schwannoma (VS) patients. While overlap in specialty focus has the potential to stimulate multidisciplinary collaboration and innovative thinking, there is a risk of specialties forming closed-communication loops, called knowledge silos, which may inhibit knowledge diffusion. This study quantitatively assessed knowledge sharing between neurosurgery and otolaryngology on the subject of VS.

METHODS A broad Web of Science search was used to download details for 4439 articles related to VS through 2016. The publishing journal's specialty and the authors' specialties (based on author department) were determined for available articles. All 114,647 of the article references were categorized by journal specialty. The prevalence of several VS topics was assessed using keyword searches of titles.
\end{abstract}

RESULTS For articles written by neurosurgeons, $44.0 \%$ of citations were from neurosurgery journal articles and $23.4 \%$ were from otolaryngology journals. The citations of otolaryngology authors included $11.6 \%$ neurosurgery journals and $56.5 \%$ otolaryngology journals. Both author specialty and journal specialty led to more citations of the same specialty, though author specialty had the largest effect. Comparing the specialties' literature, several VS topics had significantly different levels of coverage, including radiosurgery and hearing topics. Despite the availability of the Internet, there has been no change in the proportions of references for either specialty since 1997 (the year PubMed became publicly available).

CONCLUSIONS Partial knowledge silos are observed between neurosurgery and otolaryngology on the topic of VS, based on the peer-reviewed literature. The increase in access provided by the Internet and searchable online databases has not decreased specialty reference bias. These findings offer lessons to improve cross-specialty collaboration, physician learning, and consensus building.

https://thejns.org/doi/abs/10.3171/2017.6.JNS171182

KEY WORDS collaboration; development; diffusion; innovation; learning; specialty; vestibular schwannoma

$\mathrm{S}$ INCE the earliest treatment attempts for vestibular schwannomas (VS), investigators from both neurosurgery and neurotology have contributed to medical progress with respect to this tumor. ${ }^{14}$

Harvey Cushing, the father of American neurosurgery, recognized and described unilateral hearing loss and tinnitus as tumor-related symptoms, allowing earlier VS diagnosis. ${ }^{11}$ Through exacting improvements in surgical technique, Cushing and his pupil Walter Dandy drove VS resection from an intervention of last resort, with over
$80 \%$ operative mortality, to a treatment with a mortality rate as low as $10 \% .^{11}$

In the early 1960s, the father of neurotology, William House, brought the surgical microscope and the highspeed drill to VS resection for the first time. ${ }^{17}$ With these tools, he improved the translabyrinthine approach, developed the middle fossa approach, and set the field on a course to eventually reach the impressive surgical outcomes seen today. ${ }^{14}$

Rapport between neurosurgeons and neurotologists has

ABBREVIATIONS AN = acoustic neuroma; ENT = otolaryngology/otolaryngologist; NS = neurosurgery/neurosurgeon; SRS = stereotactic radiosurgery; VS = vestibular schwannoma.

SUBMITTED May 13, 2017. ACCEPTED June 19, 2017.

INCLUDE WHEN CITING Published online December 1, 2017; DOI: 10.3171/2017.6.JNS171182. 
evolved over time. Initially, when House began operating on VSs, a degree of animosity and competition defined the relationship. ${ }^{6,11}$ But over the subsequent decades, specialists became close collaborators and now frequently work as teams in the operating room. ${ }^{5,23}$

Involvement of multiple physician specialties in the treatment of the same disease offers the potential to stimulate interdisciplinary collaboration and increase diversity of thought. But there may also be a risk of specialty segregation, through publishing in different journals or using a different vocabulary, resulting in barriers to knowledge dissemination and the creation of "knowledge silos." The term knowledge silos borrows from the business management phrase "functional silos," describing a situation in which an organization's sections are insulated, running in parallel and not efficiently communicating with each other. ${ }^{7}$ Analogously, knowledge silos occur when collective knowledge, such as in the medical literature, is pursued in parallel and not effectively shared between concerned parties, such as distinct specialties.

Concerns about the presence of knowledge silos are made more pressing by trends driving increasing specialty overlap. The number of physician specialties continues to increase, ${ }^{4}$ with the American Board of Medical Specialties currently certifying physicians in over 150 general specialties and subspecialties (http://www.abms.org/memberboards/specialty-subspecialty-certificates/). Additionally, new technologies, such as endovascular interventions and radiosurgery, ${ }^{18,21}$ cross multiple specialties' customary boundaries and skill sets, which can lead to adoption by more than 1 specialty.

A better understanding of how knowledge is communicated across specialties on shared topics is essential to ensuring effective cross-disciplinary collaboration and driving consensus toward optimal care. The large volume of scientific literature published on VS by neurosurgeons and neurotologists offers a unique opportunity to investigate the existence of knowledge silos.

\section{Methods}

\section{Theoretical Basis of Methods}

To assess for the presence of knowledge silos, we focused on evaluating the peer-reviewed literature on VS. Published studies are a primary source of new information for physicians, ${ }^{13}$ informing clinical decisions and inspiring future investigations. Cumulatively, peer-reviewed literature can act as a surrogate for collective knowledge, and changes in literature can act as a proxy for community learning and acceptance. ${ }^{25}$

Peer-reviewed publications build on previous investigations, and study references act as accessible vectors pointing to the foundation of each article. For decades, citation analyses have been used to assess individual article or author impact, particularly through the impact factor and $\mathrm{h}$ index. ${ }^{1,9}$ In this study, we aim to categorize articles and references by their specialty affiliation to quantitatively assess cross-specialty knowledge sharing in the VS literature.

\section{Search Methodology}

A broad search of the Web of Science database was performed to capture available literature related to VS: "(vestibular schwannoma) OR (acoustic neuroma)" with filters for English language and Article (document type) from earliest available year through 2016. Web of Science was selected because it is a citation index and allows for assessment of detailed citation information.

Records for all articles, which included author information, addresses, title, cited references, times cited, and source, were exported to an EndNote library. Article records were then exported to text files using a custom export style.

Although using only one database is a study limitation, as no single database has all articles on every topic, assessing multiple databases would have presented practical challenges given the risks of double-counting and inconsistent formatting across resources. While not exhaustive, Web of Science represents a large and representative sample, allowing for confident conclusions.

\section{Programmatic Categorization}

A total of 4439 articles with 114,647 references were exported for analysis. An object-oriented programmatic script was written in Python programming language (composed and executed in the Jupyter Notebook environment) to extract and categorize data by article.

All unique journal and cited reference identities, including variations in abbreviations or typography, were extracted and manually labeled as a neurosurgery, otolaryngology, or "other" journal to serve as a reference library for categorization. The departmental affiliation (department, unit, division, etc.) for each corresponding author was searched and used to estimate author specialty. The corresponding author's departmental affiliation was identified for $89.8 \%$ of articles. Based on this affiliation, each article was designated as primarily a neurosurgery, otolaryngology, or "other" specialty authored article. Common "other" specialties included oncology, radiology, neurology, and pathology.

While the corresponding author's affiliation was considered the primary determinant of author specialty, all additional coauthor departments were also assessed and any article with both neurosurgery and otolaryngology authors was additionally labeled a "multispecialty" article.

To assess for specific topics and vocabulary related to VS, the title of each article was searched for the presence of keywords: "acoustic neuroma", "vestibular schwannoma", "radiosurgery" or "gamma", "hearing", "balance", "tinnitus", "middle fossa", "translabyrinthine", "retrosigmoid" or "suboccipital", "radiation therapy", "recurrent" or "recurrence", "complication", "natural history", "observation" or "conservative" or "wait."

\section{Statistical Analysis}

On completion of a post-quality control programmatic run, the following variables for each article were used for analysis: published year, title, publishing journal, journal specialty, author specialty (department), times cited, total number of references, number of neurosurgical references, number of otolaryngology references, and binary variables for each title search and if it was a multispecialty article. 


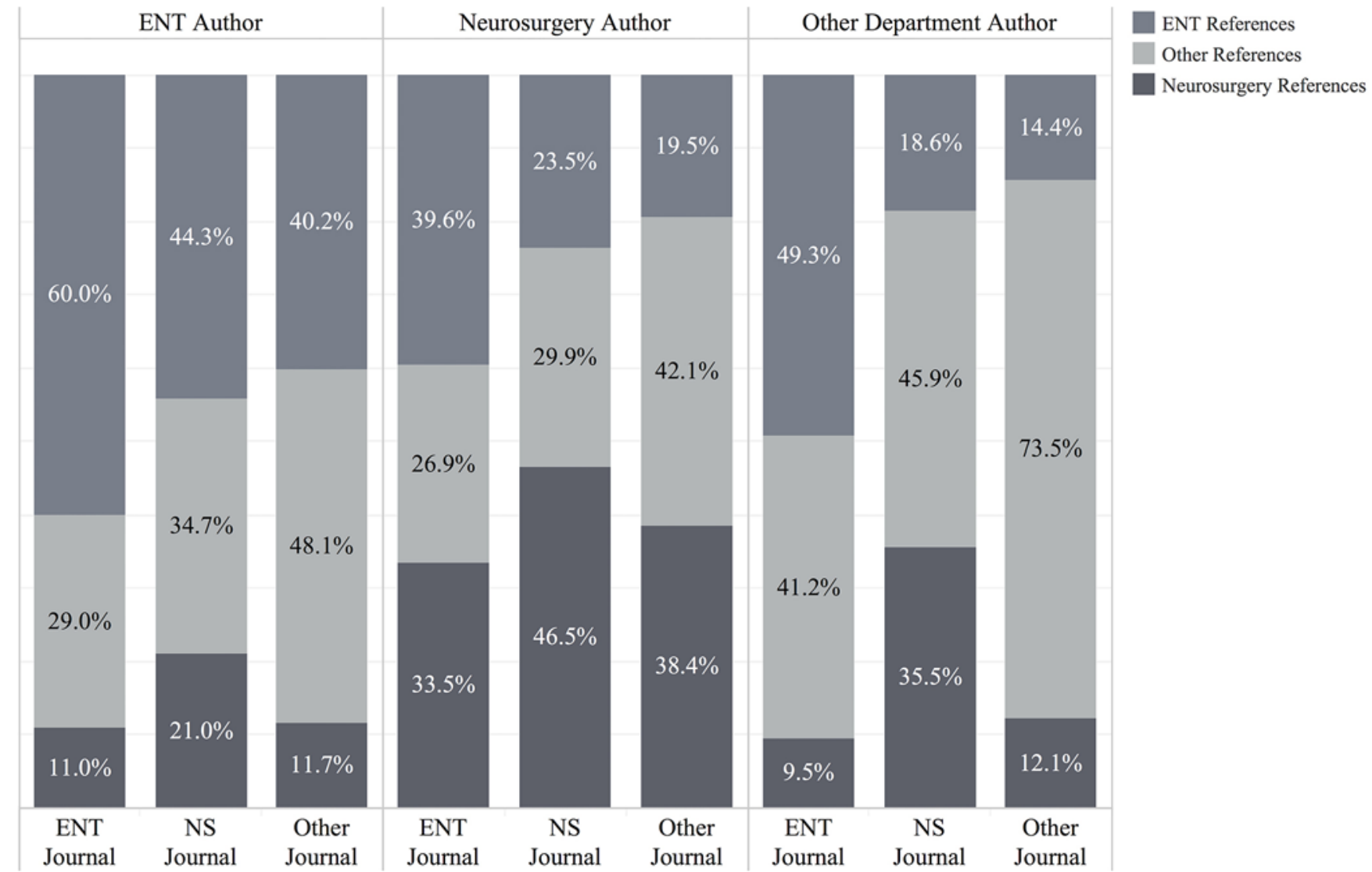

FIG. 1. Bar graph demonstrating the average specialty distribution of references by combination of author and journal specialty. Figure is available in color online only.

All analyses were performed using Stata version 14.2 (StataCorp LP). Two-sample t-tests were used to compare referencing patterns and two-sample tests of proportions were used to assess differences in topic prevalence. To assess change in reference proportions over time, linear regression was used.

\section{Results}

Of all 4439 articles on the topic of VS, 1057 (23.8\%) were published by neurosurgery (NS) journals, 2004 (45.2\%) were published by otolaryngology (ENT) journals, and 1378 (31.0\%) were published by journals with a different affiliation.

Comparing the specialty citation patterns of authors from NS departments to authors from ENT departments, NS authors had a significantly larger percentage of citations from NS journals $(44.0 \%$ vs $11.6 \%, \mathrm{p}<0.0001)$ and a smaller percentage from ENT journals $(23.4 \%$ vs $56.5 \%$, $\mathrm{p}<0.0001)$. There was no difference in the proportion of "other journals" cited by NS or ENT authors (32.6\% vs $32.0 \%, \mathrm{p}=0.5116)$.

\section{Influence of Journal Specialty and Author Specialty}

While the vast majority of authors publish in their own specialty's journals $(72.2 \%$ of NS-authored articles were in NS journals and $81.6 \%$ of ENT-authored articles were in ENT journals), there was a share of NS-authored articles published in ENT journals (5.0\% of NS-authored articles) and ENT-authored articles published in NS journals (4.3\% of ENT-authored articles).

Reference proportions based on author specialty were broken down by journal specialty to further delineate the impact of author background and journal affiliation on citation patterns (Fig. 1). Articles by authors from NS departments publishing in NS journals had the largest proportion of NS citations (46.5\%) and the lowest share of ENT citations (23.5\%). Conversely, articles by ENT authors published in ENT journals had the smallest percentage of NS citations (11.0\%) and largest share of ENT citations $(60.0 \%)$. When authors published in the other specialty's journals, the proportion of article references from their own specialty decreased and the proportion from the journal's specialty increased. For all combinations of NS/ENT authors and NS/ENT journals, the proportions of either citation group were significantly different, $\mathrm{p}<$ 0.0001 (Table 1).

When author specialty is changed, while holding journal specialty constant, there is an average swing of $22.3 \%$ of citations toward journals of the author's specialty (and away from the other specialty) (Fig. 2). Comparatively, when author specialty remains constant as the journal specialty is switched, there is an average swing of $13.7 \%$ of citations toward the specialty of the publishing journal. 
TABLE 1. Two-sample t-test results: comparison of average proportions of NS and ENT references for each combination of author and journal specialty

\begin{tabular}{|c|c|c|c|c|c|c|}
\hline \multirow{2}{*}{$\begin{array}{l}\text { Journal } \\
\text { Specialty }\end{array}$} & \multicolumn{2}{|c|}{ NS Author } & \multicolumn{2}{|c|}{ ENT Author } & \multicolumn{2}{|c|}{$p$ Value } \\
\hline & NS Refs & ENT Refs & NS Refs & ENT Refs & NS Refs & ENT Refs \\
\hline NS & $46.5 \%$ & $23.5 \%$ & $21.0 \%$ & $44.3 \%$ & $<0.0001$ & $<0.0001$ \\
\hline ENT & $33.5 \%$ & $39.6 \%$ & $11.0 \%$ & $60.0 \%$ & $<0.0001$ & $<0.0001$ \\
\hline$p$ value & $<0.0001$ & $<0.0001$ & $<0.0001$ & $<0.0001$ & & \\
\hline
\end{tabular}

\section{Multispecialty Articles}

Corresponding author affiliation was used as the primary determinant of author specialty, but articles with coauthors from both NS and ENT departments were identified and labeled as "multispecialty" articles.

When comparing the proportion of multispecialty articles, there was no difference in prevalence, irrespective of whether the primary (corresponding) author was from NS or ENT departments: 230 (20.5\%) of primarily NS-authored articles and 401 (20.9\%) of primarily ENT-authored articles had coauthors from both specialties $(p=0.8194)$.

For both groups, multispecialty articles led to an increase in the average proportion of citations of the nonprimary specialty and a decrease in the proportions of the primary author's specialty. Articles written by neurosurgeons without any ENT authors had an average of $45.9 \%$ NS citations and 19.4\% ENT citations, while articles with a primary NS author and at least 1 ENT coauthor referenced fewer NS articles $(36.6 \%, \mathrm{p}<0.0001)$ and more ENT articles $(38.8 \%, p<0.0001)$. Similarly, articles with only ENT authors had an average citation distribution of $10.0 \% \mathrm{NS}$ and $57.7 \%$ ENT compared with articles with an ENT primary author and NS coauthors, which referenced more NS articles $(17.4 \%, \mathrm{p}<0.0001)$ and fewer ENT articles $(52.0 \%, \mathrm{p}<0.0001)$. The corresponding author's departmental affiliation remained a major factor, as the reference proportions of multispecialty articles differed significantly depending on the primary author's department $(\mathrm{p}<0.0001)$.

\section{Topic Differences}

The frequencies of 12 topics related to VS, determined by keyword search of article titles, were compared for NS and ENT journals (Table 2). The prevalence of 4 topics was significantly different. Keywords related to radiosurgery occurred in the titles of $26.5 \%$ of articles published in NS journals, compared with $3.0 \%$ of articles found in ENT journals $(p<0.0001)$. The topic of "hearing" was more prevalent in ENT journals, occurring in the titles of $14.8 \%$ of ENT journal articles and $10.5 \%$ of NS journals $(\mathrm{p}=0.0008)$. For surgical approach prevalence, translabyrinthine was more frequent in ENT journals (4.7\%) than in NS journals $(1.8 \%)(\mathrm{p}<0.0001)$ and retrosigmoid/ suboccipital occurred more often in NS journals $(6.0 \%)$ than in ENT journals $(3.3 \%)(p=0.0006)$. There were no significant differences in the prevalence of the following topics: balance, tinnitus, middle fossa approach, radiation therapy, recurrent/recurrence, complications, natural history, or observation.

The use of the terms "acoustic neuroma" (AN) and "vestibular schwannoma" (VS) was also assessed. Looking at all articles in which one of the terms was used in

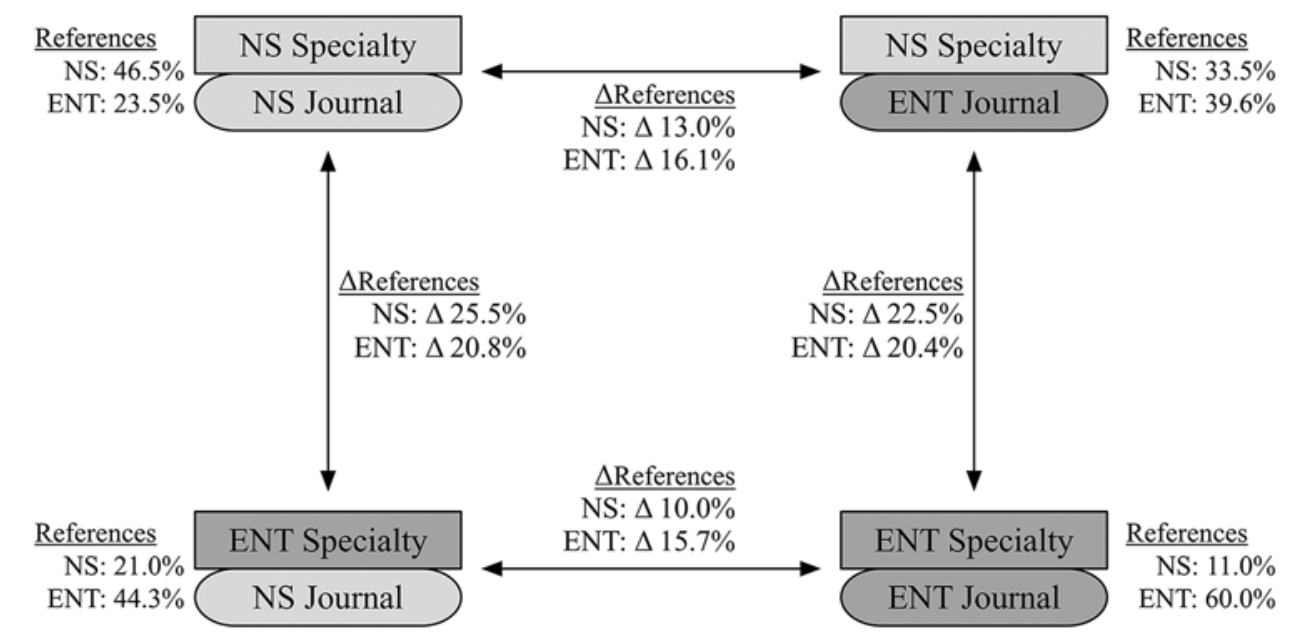

FIG. 2. Changes in specialty reference proportions with changes in author or journal specialty. Each corner represents a combination of author specialty (rectangle) and publishing journal specialty (oval) with average specialty reference proportions noted to the side. Along each arrow is shown the change in average proportions of NS and ENT references from a single change of author or journal specialty. 


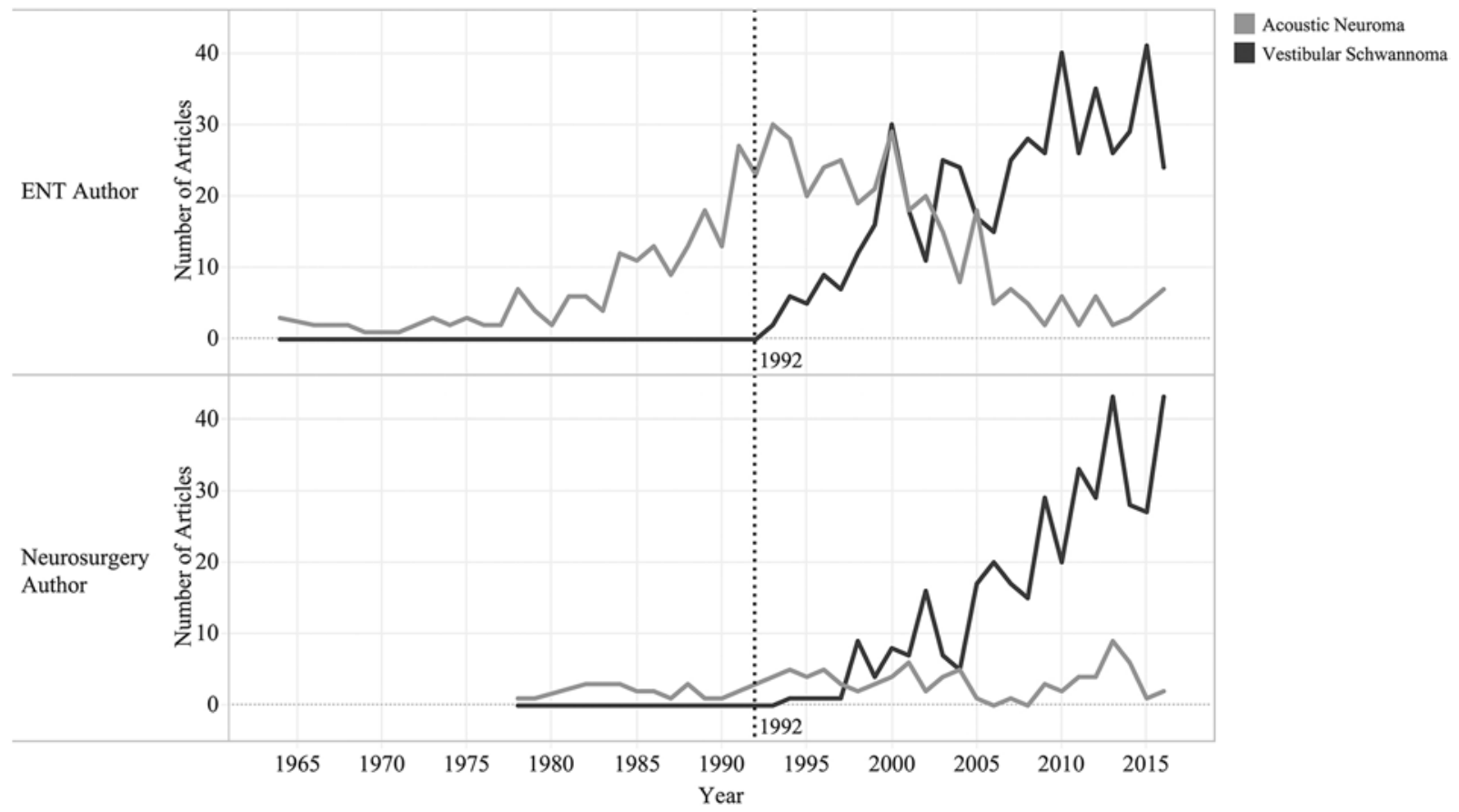

FIG. 3. Use of the terms "vestibular schwannoma" and "acoustic neuroma" over time. In 1992, the NIH Consensus Development Conference on Acoustic Neuroma issued the conclusion that "vestibular schwannoma" was the preferred term.

the title, ENT articles had a higher prevalence of AN, with $50.4 \%$ of articles using AN and $49.6 \%$ using VS, compared with NS articles, with $24.5 \%$ using AN and $75.5 \%$ using VS ( $p<0.0001)$. But this difference was mostly driven by historical usage, as can be seen in Fig. 3. Both specialties exclusively used "acoustic neuroma" until 1992, when the use of "vestibular schwannoma" began to steadily increase. AN use began to decline several years later. In articles published after 2005, there is no significant difference in the proportional use of the terms, with subsequent frequency of the term VS at $86.4 \%$ in ENT articles and $87.9 \%$ in NS articles $(\mathrm{p}=0.5180)$.

\section{Impact of the Internet}

To assess whether the Internet and the development of searchable online databases had any effect on cross-specialty referencing, we assessed the distribution of article citation over time. From 1997 (the year PubMed became widely available) through 2016, the average proportions of NS and ENT references per year remained stable for both NS and ENT authors (Fig. 4 upper). For both author groups, the difference between the proportions of NS references and ENT references demonstrated a slope very close to zero, with a slope of $0.009(\mathrm{p}<0.001)$ for ENT authors and $0.006(\mathrm{p}=0.03)$ for NS authors (Fig. 4 lower).

\section{Discussion}

In an environment where knowledge flows seamlessly between specialties, it would be expected that articles on the same subject, independent of the author's specialty or the publishing journal's affiliation, would cite relevant studies in equal proportions on average.
In this investigation, it was observed that a reference bias existed between neurosurgery and otolaryngology on the subject of VS. Authors of both specialties disproportionately cited more articles published in their own specialty's journals and fewer studies published in the opposite specialty's literature.

While the specialty of the publishing journal influenced article reference proportions, author specialty had a larger effect, independent of journal affiliation (Fig. 2). While explanations for this effect include career pressures or social relationships, the most likely explanation is that authors most frequently read, are more familiar with, and most trust articles published within their own specialty. This preference could arise from limited access, if specialists only maintain subscriptions for their specialty's journals, or it could be due to cultural or behavioral factors.

This bias toward referencing within a specialty is a barrier to knowledge diffusion that could indicate a knowledge silo. At an extreme, a complete knowledge silo is a circular, closed information loop, with each specialty pursuing knowledge independently of the other. Of course, these specialties are not wholly separate, as demonstrated by some cross-specialty referencing.

But even partial knowledge silos could be impactful. If specialties treating and researching the same subject are not in sync and information is not communicated seamlessly between them, there may be delays in the most current knowledge being reviewed, accepted, and adopted. Physicians of different specialties might then base decisions, including clinical decisions or decisions about which research directions to pursue, on different underlying information. 


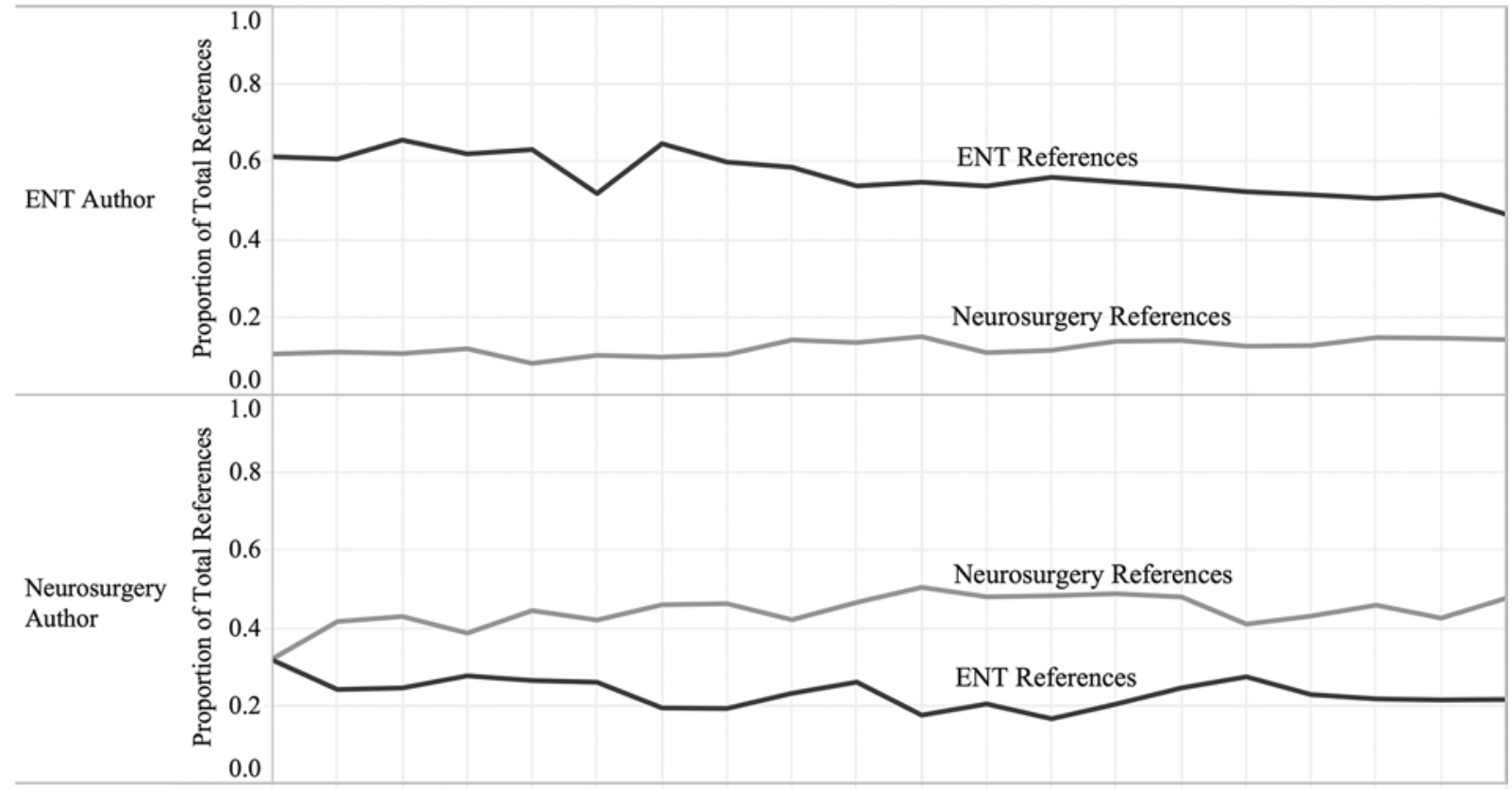

199819992000200120022003200420052006200720082009201020112012201320142015

Year

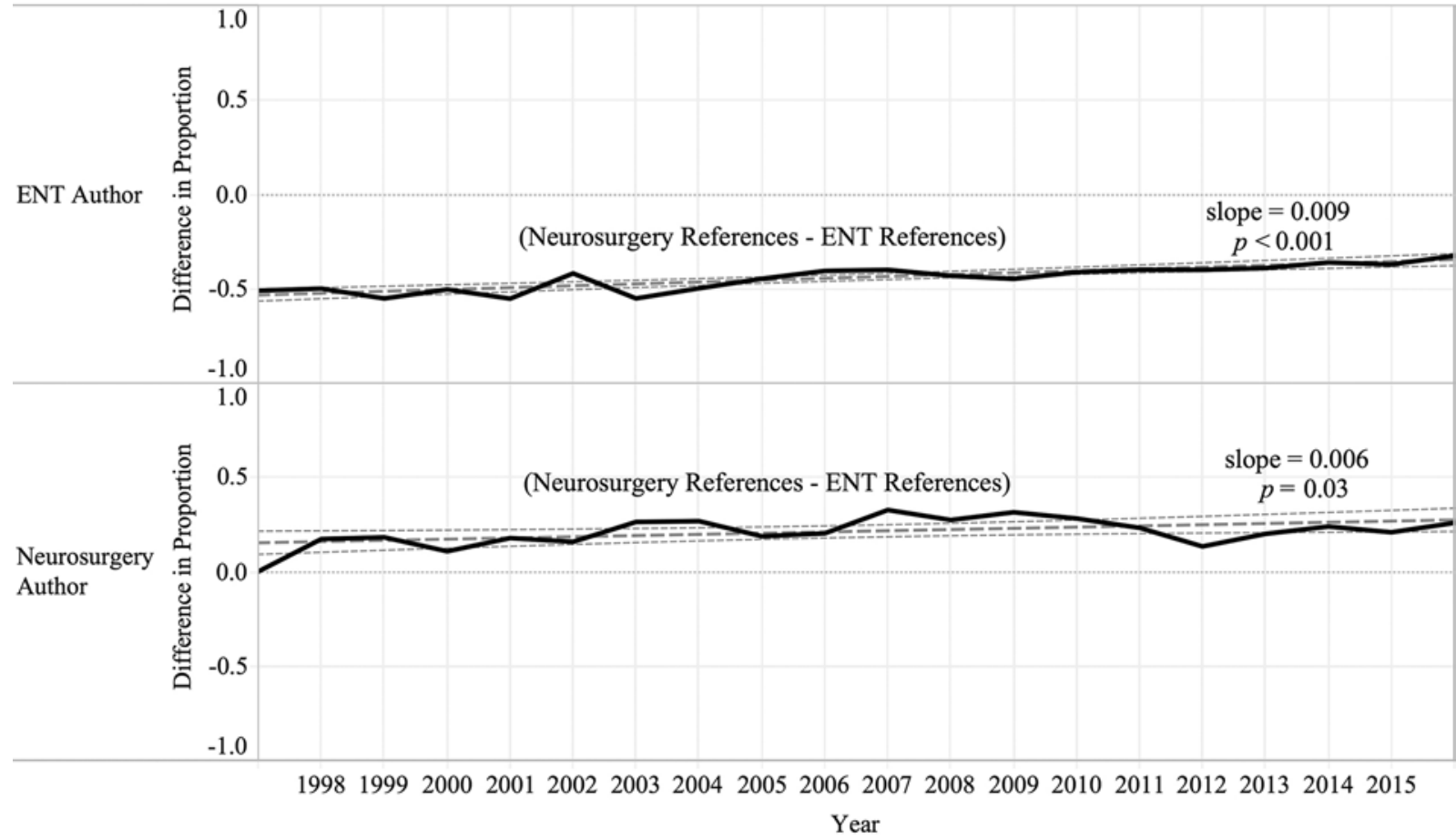

FIG. 4. Proportions of NS and ENT references over time. Upper: Average proportion of NS and ENT references per year (noncumulative) by author specialty. Lower: Difference in average proportion of NS and ENT references per year with accompanying regression line to assess trend.

\section{Topic Differences}

Assessing whether there were content differences between the 2 specialties was important in evaluating knowledge silos. If the one specialty's literature was essentially the same as the others, then a propensity to cite within a specialty would not result in different conclusions and might not be consequential.
When the prevalence of VS topics in the publications of each specialty was assessed, there were significant differences (Table 2). Radiosurgery was a topic in $26.5 \%$ of neurosurgery journal articles but only in $3.0 \%$ of articles in ENT publications. Conversely, articles related to hearing were significantly less common in the neurosurgical press than in the ENT literature. Given the importance 
of hearing topics and the demonstrated efficacy and firstline considerations of stereotactic radiosurgery (SRS) for $\mathrm{VS},{ }^{15,16,20}$ these differences raise questions about learning and clinical practices.

The field of adult learning has greatly informed understanding of continuing medical education and physician learning. Self-directed learning proceeds through 3 stages: 1) awareness of the need to learn, 2) learning the new skills or insights, and 3) identifying how to incorporate new competencies., ${ }^{3,8}$ The learning resources most frequently used by physicians, influencing all 3 stages, are medical journals, colleagues, and formal continuing education programs. ${ }^{8}$ Less coverage of a topic from a specialty's journals could result in less exposure and a hindrance to learning, including not realizing the need to search for information on a newer topic.

A major concern is that differences in coverage could contribute to differences in care. One previous study found that the attending's specialty was the strongest predictor of VS treatment choice. ${ }^{22}$ In addition to patient-specific information, many factors contribute to physician treatment preferences, including training, prior experience, and even geographical location. ${ }^{2}$ But since the medical literature is a vital part of physician learning and a major source for researching clinical questions, ${ }^{8,13}$ it is plausible that variations in topic coverage contribute to or perpetuate specialty differences in clinical decision making. Additional research is necessary to objectively assess the link between differences in specialty literature and treatment preferences.

\section{Impact of the Internet}

These findings indicate a need for solutions to further enhance knowledge sharing. Technological solutions to increase access to articles from all specialties are one consideration. In 1997, PubMed launched publicly, ${ }^{19}$ allowing free searching of the MEDLINE database from any computer with Internet access. The proportion of physicians using the Internet expanded from $10 \%$ in 1997 to $70 \%$ by 2000 to virtually $100 \%$ by $2002 .^{3}$

These advances offered unprecedented access to medical science. We expected that increased access to articles from other specialty journals would result in a convergence of reference proportions. Surprisingly, there has been no substantial change since the advent of PubMed in the citation patterns from either specialty (Fig. 4). While it is possible that use of medical databases has not fully penetrated physician practice or that journal paywalls continue to hinder access, a trend toward convergence would still be expected if access were the principle driver of citation differences.

This finding indicates that knowledge silos are not driven solely by access. It underscores that other factors, possibly cultural or behavioral, contribute to physician bias toward their own specialty's literature.

\section{Considering Solutions and Challenges}

Given time and cost constraints, it is impractical for any person to read all specialty journals related to a topic of interest. ${ }^{12}$ This creates a challenge for individuals trying to stay informed beyond their specialty's literature. One strategy is to activate automatic updates from databases,
TABLE 2. Comparison of topic prevalence between NS journals and ENT journals on topic of VS

\begin{tabular}{lrrr}
\hline \multicolumn{1}{c}{ Topic } & NS Journals & ENT Journals & p Value \\
\hline Radiosurgery & $26.5 \%$ & $3.0 \%$ & $<0.0001$ \\
\hline Hearing & $10.5 \%$ & $14.9 \%$ & 0.0007 \\
\hline Balance & $0.3 \%$ & $0.5 \%$ & 0.3015 \\
\hline Tinnitus & $0.6 \%$ & $1.3 \%$ & 0.0591 \\
\hline Middle fossa & $1.4 \%$ & $2.0 \%$ & 0.2534 \\
\hline Translabyrinthine & $1.8 \%$ & $4.7 \%$ & $<0.0001$ \\
\hline Retrosigmoid/suboccipital & $6.0 \%$ & $3.3 \%$ & 0.0006 \\
\hline Radiation therapy & $0.3 \%$ & $0.3 \%$ & 0.9397 \\
\hline Recurrent/recurrence & $0.9 \%$ & $1.1 \%$ & 0.6077 \\
\hline Complication & $1.9 \%$ & $1.5 \%$ & 0.4124 \\
\hline Natural history & $0.4 \%$ & $0.4 \%$ & 0.9303 \\
\hline Observation & $2.3 \%$ & $2.7 \%$ & 0.4319 \\
\hline Topic prevalnce was &
\end{tabular}

Topic prevalence was assessed as the percentage of each specialty's journal articles with topic keywords in title.

including PubMed, to provide email updates with new results on previously designated search topics (https://www. ncbi.nlm.nih.gov/books/NBK53592/). Yet there remains a need for additional content management tools that can effectively convert the overwhelming volume of scientific literature into useful, curated article collections on relevant topics that span journals and specialties. One barrier to development of such tools is that content is spread across a large number of proprietary sites, ${ }^{27}$ and future efforts will be necessary to coordinate across subscription journals.

Another difficulty involves the cyclical nature of knowledge silos. If one specialty publishes on a topic more frequently, it may signal to authors that related future findings fit best at those publications, further increasing the topic prevalence for those journals. Journal editors and reviewers may be able to break this cycle by remaining open to topics in which their specialty is inherently deficient.

Hospital administrative restrictions may also contribute to specialty differences in research and treatment decisions. Neurosurgeons were the developers and earliest adopters of SRS, ${ }^{21}$ adding the technique to their choices for management. While some neurotologists are treating VS patients with SRS, performance requires training, access, and local credentialing. ${ }^{10}$

Traditional efforts to build consensus among stakeholders offer additional lessons about potential solutions. A notable collaborative effort was the 1992 NIH Consensus Development Conference on Acoustic Neuroma, which brought together leaders from multiple specialties and professionals concerned with VS to reach agreement on several issues. $^{21}$

One conclusion of the panel was that "vestibular schwannoma" was the preferred term for the tumor, given it is composed of Schwann cells and arises most frequently from the vestibular branch of the eighth cranial nerve. ${ }^{21}$ The impact of this announcement can be seen in changes in the literature (Fig. 3). After the NIH meeting in 1992, there was an increase in the use of the term vestibular schwannoma by neurosurgery and otolaryngology authors until it became 
the dominant term used by both groups. While replicating this pattern may be more challenging for more controversial issues, this meeting offers a notable example of consensus building. Both new solutions and traditional strategies may be necessary to increase cross-specialty consensus.

\section{Conclusions}

In this study, we quantitatively assessed knowledge sharing between neurosurgery and otolaryngology on the subject of VS. Through assessment of the peer-reviewed literature, we identified partial knowledge silos dividing specialties, with significant differences in VS topic coverage and biases toward referencing within the same specialty. The Internet and the establishment of online medical databases have not changed reference biases. Despite limitations, these findings offer several important lessons for increasing collaboration, improving physician learning, and driving cross-specialty consensus. Future investigations assessing the impact of knowledge silos on clinical and research decisions are necessary.

\section{Acknowledgments}

Zane Schnurman received funding for this work from NYU School of Medicine's Rudin Medical Ethics and Humanities Fellowship.

\section{References}

1. Braun T, Glanzel W, Schubert A: A Hirsch-type index for journals. Scientometrics 69:169-173, 2006

2. Carlson ML, Glasgow AE, Grossardt BR, Habermann EB, Link MJ: Does where you live influence how your vestibular schwannoma is managed? Examining geographical differences in vestibular schwannoma treatment across the United States. J Neurooncol 129:269-279, 2016

3. Casebeer L, Bennett N, Kristofco R, Carillo A, Centor R: Physician Internet medical information seeking and on-line continuing education use patterns. J Contin Educ Health Prof 22:33-42, 2002

4. Cassel CK, Reuben DB: Specialization, subspecialization, and subsubspecialization in internal medicine. $\mathbf{N}$ Engl J Med 364:1169-1173, 2011

5. Darrouzet V, Martel J, Enée V, Bébéar JP, Guérin J: Vestibular schwannoma surgery outcomes: our multidisciplinary experience in 400 cases over 17 years. Laryngoscope 114:681-688, 2004

6. DiTullio MV Jr, Malkasian D, Rand RW: A critical comparison of neurosurgical and otolaryngological approaches to acoustic neuromas. J Neurosurg 48:1-12, 1978

7. Ensor PS: The functional silo syndrome. AME Target 16:16, 1988

8. Fox RD, Bennett NL: Learning and change: implications for continuing medical education. BMJ 316:466-468, 1998

9. Garfield E: Citation indexes for science; a new dimension in documentation through association of ideas. Science 122:108-111, 1955

10. German MA, Zardouz S, Sina MK, Ziai K, Djalilian HR: Stereotactic radiosurgery for vestibular schwannomas: a survey of current practice patterns of neurotologists. Otol Neurotol 32:834-837, 2011

11. Glasscock ME III: History of the diagnosis and treatment of acoustic neuroma. Arch Otolaryngol 88:578-585, 1968
12. Greenhalgh T, Howick J, Maskrey N: Evidence based medicine: a movement in crisis? BMJ 348:g3725, 2014

13. Haug JD: Physicians' preferences for information sources: a meta-analytic study. Bull Med Libr Assoc 85:223-232, 1997

14. Koerbel A, Gharabaghi A, Safavi-Abbasi S, Tatagiba M, Samii M: Evolution of vestibular schwannoma surgery: the long journey to current success. Neurosurg Focus 18(4):e10, 2005

15. Kondziolka D, Lunsford LD, McLaughlin MR, Flickinger JC: Long-term outcomes after radiosurgery for acoustic neuromas. N Engl J Med 339:1426-1433, 1998

16. Kondziolka D, Mousavi SH, Kano H, Flickinger JC, Lunsford LD: The newly diagnosed vestibular schwannoma: radiosurgery, resection, or observation? Neurosurg Focus 33(3):E8, 2012

17. Kriss TC, Kriss VM: History of the operating microscope: from magnifying glass to microneurosurgery. Neurosurgery 42:899-908, 1998

18. Lakhan SE, Kaplan A, Laird C, Leiter Y: The interventionalism of medicine: interventional radiology, cardiology, and neuroradiology. Int Arch Med 2:27, 2009

19. Lindberg DA: Internet access to the National Library of Medicine. Eff Clin Pract 3:256-260, 2000

20. Myrseth E, Møller P, Pedersen PH, Lund-Johansen M: Vestibular schwannoma: surgery or gamma knife radiosurgery? A prospective, nonrandomized study. Neurosurgery 64:654-663, 2009

21. National Institutes of Health: Acoustic neuroma. NIH Consens Statement 9:1-24, 1991

22. Niranjan A, Madhavan R, Gerszten PC, Lunsford LD: Intracranial radiosurgery: an effective and disruptive innovation in neurosurgery. Stereotact Funct Neurosurg 90:1-7, 2012

23. Pogodzinski MS, Harner SG, Link MJ: Patient choice in treatment of vestibular schwannoma. Otolaryngol Head Neck Surg 130:611-616, 2004

24. Saeed SR, Suryanarayanan R, Dezso A, Ramsden RT: Vestibular schwannoma management: current practice amongst UK otolaryngologists-time for a national prospective audit. Ann R Coll Surg Engl 88:490-495, 2006

26. Schnurman Z, Kondziolka D: Evaluating innovation. Part 1: The concept of progressive scholarly acceptance. J Neurosurg 124:207-211, 2016

27. Sedory Holzer SE, Kokemueller P: Internet platforms for lifelong learning: a continuum of opportunity. Otolaryngol Clin North Am 40:1275-1293, viii, 2007

\section{Disclosures}

The authors report no conflict of interest concerning the materials or methods used in this study or the findings specified in this paper.

\section{Author Contributions \\ Conception and design: all authors. Acquisition of data: Schnur- man. Analysis and interpretation of data: all authors. Drafting the article: all authors. Critically revising the article: all authors. Reviewed submitted version of manuscript: all authors. Approved the final version of the manuscript on behalf of all authors: Kond- ziolka. Statistical analysis: all authors. Administrative/technical/ material support: Kondziolka. Study supervision: Kondziolka.}

\section{Correspondence}

Douglas Kondziolka, Department of Neurosurgery, NYU Langone Medical Center, 530 First Ave., Ste. 8R, New York, NY 10016. email: douglas.kondziolka@nyumc.org. 